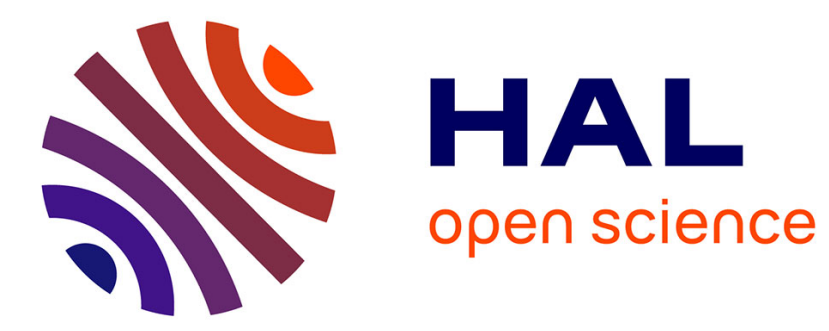

\title{
Etude du stockage de l'oxygène sur des catalyseurs modèles de post-combustion automobile
}

\author{
Sumeya Bedrane, Claude Descorme, Daniel Duprez
}

\section{To cite this version:}

Sumeya Bedrane, Claude Descorme, Daniel Duprez. Etude du stockage de l'oxygène sur des catalyseurs modèles de post-combustion automobile. Journal de Physique IV Proceedings, 2002, 12, pp.1523. $10.1051 /$ jp420020010 . hal-00291135

\section{HAL Id: hal-00291135 \\ https://hal.science/hal-00291135}

Submitted on 12 Oct 2021

HAL is a multi-disciplinary open access archive for the deposit and dissemination of scientific research documents, whether they are published or not. The documents may come from teaching and research institutions in France or abroad, or from public or private research centers.
L'archive ouverte pluridisciplinaire HAL, est destinée au dépôt et à la diffusion de documents scientifiques de niveau recherche, publiés ou non, émanant des établissements d'enseignement et de recherche français ou étrangers, des laboratoires publics ou privés. 


\title{
Étude du stockage de l'oxygene sur catalyseurs modeles de post-combustion automobile
}

\author{
S. Bedrane, C. Descorme et D. Duprez \\ Laboratoire de Catalyse en Chimie Organique, LACCO, UMR 6503 du CNRS, \\ Universite de Poitiers, Faculte des Sciences, 40 avenue du Recteur Pineau, \\ 86022 Poitiers cedex, France
}

\begin{abstract}
Resume: Différents catalyseurs modèles de post-combustion automobile ont été étudiés. Ces échantillons sont du type métaux précieux ( $\mathrm{Rh}$, Pt et $\mathrm{Pd})$ supportés sur cérine $(\mathrm{CeO})$ et cérine-zircone $\left(\mathrm{Ce}_{0,63} \mathrm{Zr}_{0,3} \mathrm{O}_{2}\right)$. Les catalyseurs ont été caractérisés par diffraction des rayons-X, chimisorptionde l'hydrogène et adsorption de l'azote à $-196^{\circ} \mathrm{C}$. Le stockage de l'oxygène dans ces matériaux a été largement étudié. L'influence de divers paramètres a été analysée tels que : la nature du métal, la morphologie des particules métalliques, le support et la température.
\end{abstract}

\section{INTRODUCTION}

La protection de notre environnement constitue aujourd'hui un enjeu majeur. En effet, les problèmes decoulant de l'augmentation de la pollution atmosphérique apparaissent comme étant de plus en plus nombreux et sévères. Le secteur des transports contribue pour une part importante a la deterioration de la qualité de l'air. Ainsi, l'accroissement du trafic routier, principalement dans les grandes agglomkrations, est a l'origine de serieux problèmes de santé publique. Une prise de conscience générale de I'impact de la pollution automobile a mene les autoritks compétentes, nationales et européennes, a mettre en place des normes fixant les limites maximales des emissions de gaz toxiques tels que : le monoxyde de carbone (CO), les hydrocarbures (HC) et les oxydes d'azote (NO,). Ainsi, depuis l'entrée en vigueur des normes anti-pollution en 1970, l'émission des diffkrents polluants a diminué de $96 \%$. Les recherches actuelles portent sur l'élimination des derniers $4 \%$. En parallèle, d'importants efforts ont été effectués avec la mise en place d'essences "légères" en plomb et en soufre, l'amélioration de la combustion dans les moteurs et le développement de "pots catalytiques" pour le post-traitement des gaz d'échappement.

Un convertisseur catalytique est constitué d'une phase active - du type métaux précieux (Rh, $\mathrm{Pt}, \mathrm{Pd})$ dispersée sur un support de grande surface spécifique (le washcoat) recouvrant les parois d'un monolithe en cordiérite $\left(2 \mathrm{MgO}, 2 \mathrm{Al}_{2} \mathrm{O}_{3}, 5 \mathrm{SiO}_{2}\right)$ en forme de nids d'abeille.

Dans le cas d'un vkhicule essence a allumage commandé, ce catalyseur est dit "trois voies" (TWC) puisqu'il doit simultanément oxyder le monoxyde de carbone $(\mathrm{CO})$ et les hydrocarbures imbrûlés $(\mathrm{HC})$ en $\mathrm{CO}_{2}$ :

$\mathrm{CO}+\mathrm{O}_{2} \rightarrow \mathrm{CO}_{2}$

$\mathrm{HC}+\mathrm{O}_{2} \rightarrow \mathrm{CO}_{2}+\mathrm{H}_{2} \mathrm{O}$ 
et reduire les $\mathrm{NO}$, en $\mathrm{N}_{2}$

$\mathrm{NO}+\mathrm{CO} \rightarrow \mathrm{N}_{2}+\mathrm{CO}_{2}$

$\mathrm{NO}+\mathrm{H}_{2} \rightarrow \mathrm{N}_{2}+\mathrm{H}_{2} \mathrm{O}$

$\mathrm{NO}+\mathrm{HC} \rightarrow \mathrm{N}_{2}+\mathrm{CO}_{2}$

Ces réactions d'oxydation et de reduction n'ont lieu en même temps avec un rendement optimum que lorsque les gaz d'echappement correspondent a un melange stœechiométrique contenant des quantités equivalentes d'oxydants $\left(\mathrm{O}_{2}, \mathrm{NO}\right.$, ) et de réducteurs $\left(\mathrm{CO}, \mathrm{HC}, \mathrm{H}_{2}\right)$, c'est-a-dire pour un rapport air/carburant de 14,6 (Figure 1).

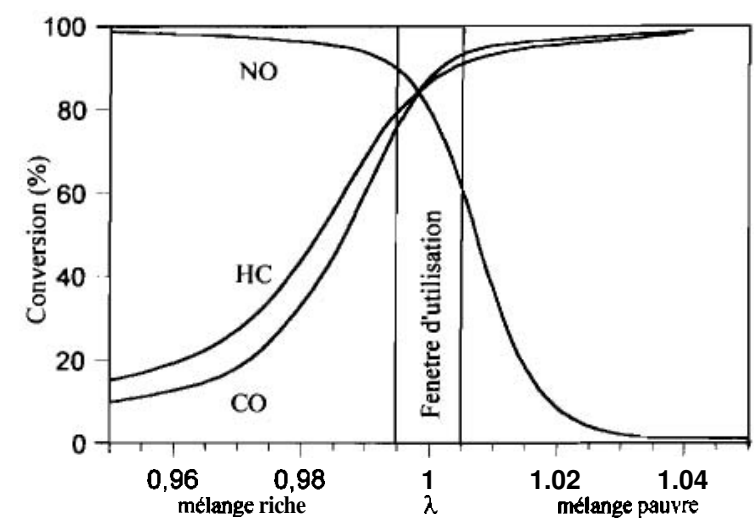

Figure 1 - Conversion de CO, HC et NO, en fonction du rapport air/carburant (k)

Toutefois, en fonctionnement reel, la composition des gaz d'echappement oscille autour de la stœchiométrie en alternant phases riches (defaut d'oxygene) et phases pauvres (excts d'oxygtne). Aussi, pour obtenir une conversion optimale des 3 polluants, il est necessaire d'elargir la "fenêtre d'utilisation" des catalyseurs trois-voies. C'est la raison de l'utilisation de la cerine $\left(\mathrm{CeO}_{2}\right)$ dans ces systemes catalytiques [1]. En effet, du fait de la facilité et de la rapidite de basculement du couple redox $\mathrm{Ce}^{3+} / \mathrm{Ce}^{4+}$, la cerine peut stocker l'oxygtne pendant les phases pauvres en carburant et restituer l'oxygene stocké pendant les phases riches.

Cependant, en regime transitoire, la formation de $\mathrm{Ce}_{2} \mathrm{O}_{3}$ - correspondant a une reduction complete de la cerine - n'est pas observée et la reduction de la cerine s'arrête a la formation d'un oxyde nonstœchiométrique $\mathrm{CeO}_{2-\mathbf{x}}$. Les reactions regissant le stockage (7) et le destockage (6) de l'oxygene s'ecrivent alors:

$\mathrm{CeO}_{2}+\mathrm{x} \mathrm{CO} \rightarrow \mathrm{CeO}_{2-x}+\mathrm{x} \mathrm{CO}_{2}$

$\mathrm{CeO}_{2-\mathrm{x}}+\frac{1}{2} \mathrm{xO}_{2} \rightarrow \mathrm{CeO}_{2}$

Cette propriete de la cerine a stocker et a restituer l'oxygene peut être quantifiee par la mesure de sa capacité de stockage de l'oxygene appelte OSC (Oxygen Storage Capacity).

Yao et Yu Yao [2] ont ete les premiers a mettre au point une methode pour mesurer l'OSC des oxydes. La technique consiste a réduire $\mathrm{CeO}_{2}$ sous $\mathrm{CO}$ (restitution de l'oxygene) et a la re-oxyder sous $\mathrm{O}_{2}$ (stockage de I'oxygene). Une telle mesure permet de dtterminer la quantité maximale d'oxygene pouvant être stocke sur le materiau (OSCC) ainsi que la quantité d'oxygtne immédiatement disponible (OSC). Selon ces auteurs, l'oxygene peut Ctre stocke dans la cerine sous forme d'anions de deux types differents: un anion de surface lié à un $\mathrm{Ce}^{4+}$ dans une coordination octaédrique et un anion d'oxygène lié à deux $\mathrm{Ce}^{4+}$ au cœur de la cerine. 
Depuis ces travaux, de nombreuses recherches ont ete dediees a l'étude de I'OSC des materiaux a base de cérine [3-7]. Dernièrement, la cérine est remplacée par des oxydes mixtes cérine-zircone de formules $\mathrm{Ce}_{\mathrm{x}} \mathrm{Zr}_{1-\mathrm{x}} \mathrm{O}_{2}$. Ces materiaux présentent une meilleure capacité de stockage en oxygène doublée d'une stabilité thermique accrue [8]. Un optimum de I'OSC est observe pour les oxydes mixtes riches en cerium (20 a $40 \%$ en rnasse de $\mathrm{ZrO}_{2}$ ) [9].

En dépit de la complexité de la formulation des catalyseurs trois-voies, une corrélation directe a pu être etablie entre I'OSC et la stabilité thermique d'une part et les performances catalytiques d'autre part. Aussi, l'amélioration de l'efficacité des catalyseurs et la prévention de la désactivation passe par une meilleure comprehension des processus de stockage de l'oxygène. Pow cela, notre recherche s'est orientée vers l'étude de catalyseurs de métaux nobles supportés sur cérine et cérine-zircone. Ces materiaux ont été finement caractérisés a l'aide de diverses techniques de caractérisation physicochimiques. Enfin, la capacité de stockage en oxygène de ces solides a été mesurée et l'influence de divers paramètres a été analysée.

\section{PARTIE EXPERIMENTALE}

Un catalyseur mktallique est constitué d'îlots métalliques dispersés sur un support. En catalyse, la reaction se produit a la surface du matériau. Ainsi, seuls les atomes de metal accessibles en surface participent a la reaction. Les métaux supportes devront donc être aussi finement divisés que possible, augmentant de fait le rapport entre le nombre d'atome de surface et le nombre d'atome de volume. Le rôle du support est alors de disperser la phase métallique de manière optirnale de façon a avoir un maximum d'atomes de metal en surface. En catalyse toujours, le rapport entre le nombre d'atomes en surface sur le nombre total d'atomes de metal définit la dispersion metallique ou encore la fraction métallique exposée. La dispersion, inversement proportionnelle à la taille des particules, dépendra a la fois de la methode de preparation, du sel précurseur du metal, de la nature du prétraitement de l'échantillon ...

\subsection{Préparation des catalyseurs}

L'oxyde de cérium $\mathrm{CeO}_{2}$ et I'oxyde mixte $\mathrm{Ce}_{0,63} \mathrm{Zr}_{0,37} \mathrm{O}_{2}$ nous ont été fournis par Rhodia Electronics and Catalysis (La Rochelle, France). Ces oxydes ont été préalablement calcines a $900^{\circ} \mathrm{C}$ pendant 6 heures. Les métaux précieux ( $R h, P_{t}, \mathrm{Pd}$ ) ont été deposes sur les supports par impregnation a temperature ambiante a partir d'une solution aqueuse du sel du précurseur métallique $\left(\mathrm{Rh}\left(\mathrm{NO}_{3}\right)_{3}, \mathrm{Pt}\left(\mathrm{NH}_{3}\right)_{4}(\mathrm{OH})_{2}\right.$ et $\mathrm{Pd}\left(\mathrm{NO}_{3}\right)_{3}$ respectivement). Les catalyseurs ont été prepares de façon à ce que les catalyseurs soient a isoteneur atomique en métal, soit 100 micromoles d'atomes de metal par gramme de catalyseur. Apres impregnation, les catalyseurs ont été séchés $24 \mathrm{~h}$ a $120^{\circ} \mathrm{C}$ puis traités pendant $4 \mathrm{~h}$ à $500^{\circ} \mathrm{C}\left(0.5^{\circ} \mathrm{C} . \mathrm{min}^{-1}\right)$ sous flux d'air $\left(30 \mathrm{~cm}^{3} \cdot \mathrm{min}^{-1}\right)$. La composition finale des catalyseurs après prétraitement a été obtenue par analyse chimique élémentaire (Service Central d' Analyse du CNRS de Solaize, France).

\subsection{Caractérisation}

La structure cristallographique des materiaux utilisés a été déterminée par diffraction des rayons X sur un diffractomètre de poudre Siemens D5005. Les différentes phases cristallines ont été identifiées par comparaison du diffractogramme expérimental avec les fiches ICDD (International Center for Diffraction Data). La taille moyenne des cristallites a été estimée a partir de l'élargissement des pics de diffraction selon la relation de Debye-Schemer (8).

$$
\mathrm{L}_{\mathrm{hkl}}=\frac{\lambda}{\beta \cos \theta}
$$


avec : - $\mathrm{L}_{\mathrm{hkl}}$ : taille de la cristallite dans la direction perpendiculaire aux plans réflecteurs hkl

- $\lambda$ : longueur d'onde du faisceau incident

- $\beta$ : largeur intégrale du pic de diffraction (surface totale du pic divisée par l'intensité au maximum du pic de diffraction)

- $\theta$ : angle de Bragg au maximum du pic de diffraction

La texture des solides a été analysée par adsorption d'azote a la tempkrature de l'azote liquide ($\left.196^{\circ} \mathrm{C}\right)$. Les isothermes d'adsorption-désorption sont interprétées selon la méthode développée par Brunauer, Emmett et Teller (BET). Dans cette etude, nous nous sommes limités a la determination de la surface spécifique des matériaux (méthode a un point). Les mesures ont été effectuées sur des echantillons d'environ $250 \mathrm{mg}$ préalablement dégazé pendant 1 heure a $350^{\circ} \mathrm{C}$ sous un mélange à $30 \%$ d'azote dans l'hélium.

La dispersion ou fraction métallique exposée a été mesurée par chiiisorption de l'hydrogène a . $85^{\circ} \mathrm{C}$. La mesure est réalisée dans un réacteur chrornatographique pulsé. Les échantillons, d'environ 250 $\mathrm{mg}$, sont préalablement réduits sous hydrogene pendant 1 heure a $400^{\circ} \mathrm{C}$ puis dégazés 3 heures sous argon a la même tempkrature.

\subsection{Capacitd de stockage en oxygène (OSC)}

La capacité de stockage en oxygène a été mesurée selon la méthode mise au point par Yao et Yu Yao [2] et adaptée par Kacimi et al. [10]. Elle consiste à suivre I'oxydation du monoxyde de carbone (CO) en régime transitoire en l'absence d'oxygène en phase gaz (oxydation anaérobie). La quantité d'oxygene "disponible" au sein de l'oxyde est donné par la quantité de $\mathrm{CO}_{2}$ form6 au cours de la réaction :

$\mathrm{CO}_{(\mathrm{gaz})}+\mathrm{O}_{\mathrm{s}} \rightarrow \mathrm{CO}_{2(\mathrm{gaz})}+\square_{\mathrm{s}}$

La mesure est réalisée dans un réacteur chromatographique pulsé. L'échantillon de 5 à $10 \mathrm{mg}$ est introduit dans réacteur en $U$ a lit fixe puis prétraité in situ : montee en temperature sous flux d'hélium (30 $\left.\mathrm{cm}^{3} \cdot \mathrm{min}^{-1}\right)$ jusqu'a la tempkrature de mesure $\left(200 \leq \mathrm{T} \leq 500^{\circ} \mathrm{C}\right)$ et oxydation sous pulses d'oxygene. La sequence de mesure est schématisée sur la Figure 2.

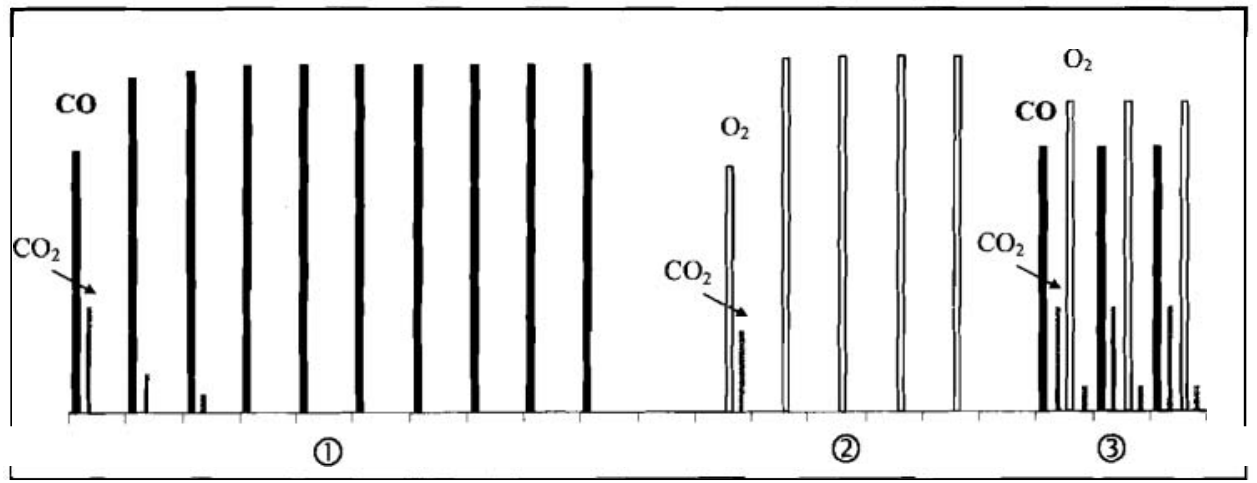

Figure 2 - Principe de la mesure de la capacité de stockage en oxygène

Apres le prétraitement, l'expérience procède comme suit :

- reduction sous pulses de CO injectés toutes les deux minutes jusqu'a saturation (1)),

- dégazage pendant 10 minutes sous flux d'hélium

- re-oxydation sous pulses d'oxygene toutes les deux minutes jusqu'a saturation (O) 
La quantité totale de $\mathrm{CO}_{2}$ formé sous pulses de $\mathrm{CO}$ permet d'accéder à la mesure de la quantité totale d'oxygene pouvant être stocké sur l'échantillon (OSCC).

A la suite de cette première etape, l'échantillon est de nouveau dégazé pendant 10 ninutes sous flux d'hélium $\left(30 \mathrm{~cm}^{3} \cdot \mathrm{min}^{-1}\right)$ puis soumis à une nouvelle série de pulses alternés $\mathrm{CO}$ ou $\mathrm{O}_{2}$ injectés toutes les de w minutes (Q). La capacité de stockage en oxygène (OSC) est déterminée a partir de la quantité de $\mathrm{CO}_{2}$ formé après le prenuier pulse de $\mathrm{CO}$ de I'alternance.

\section{RESULTATS ET DISCUSSION}

\subsection{Caractérisation physico-chimigue des matérianx}

Les principales caracléristiques des oxydes sont rassemblées dans le tableau 1.

Tableau 1 - Propsiétés souctursles des orydes $\mathrm{CeO}_{2}$ et $\mathrm{Ce}_{0,62} \mathrm{Z}_{0,5} \mathrm{O}_{2}$

\begin{tabular}{ccccc}
\hline Oxydes & $\begin{array}{c}\mathrm{S}_{\mathrm{BET}} \\
\left(\mathrm{m}^{2} \cdot \mathrm{g}^{-1}\right)\end{array}$ & structure & $\begin{array}{c}\text { Parametre de } \\
\text { maille a }(\AA)\end{array}$ & $\begin{array}{c}\text { Taille de } \\
\text { cristallites }(\AA)\end{array}$ \\
\hline $\mathrm{CeO}_{2}$ & 24 & cubique & 5,4113 & 200 \\
$\mathrm{Ce}_{0,63} \mathrm{Zr}_{0,37} \mathrm{O}_{2}$ & 43 & cubique & 5,3044 & 70 \\
\hline
\end{tabular}

La cérine $\mathrm{CeO}_{2}$ est un oxyde de stucture cubique type fluorine. L'introduction d'atomes de $\mathrm{Zr}$ dans la maille de la cerine entraine une contraction de la maille. En effet les atomes de zirconium ont un rayon ionique plus faible que celui du cérium $\left(R_{Z_{r}}{ }^{4+}=0,84 \AA\right.$ et $\left.R_{C e}{ }^{4+}=0,97 \AA\right)$. Ceci se traduit par une diminution du paramètre de maille. Les diffractogramesnes confiment tzalement la fonnation d'une solution solide monophasique. Par ailleurs nous constatons que la taille moyenne des cristallites de cerine-zircone est 3 fois plus petite que la taille des cristallites de cérine.

D'aure part, les mesures de chimisorption de l'hydrogène démontrent une bonne dispersion de la phase métallique. La taille des particules de metal varie entre 10 et $15 \mathrm{~A}$ (Tableau 2). Nous constatons de manière générale que le support cérine est plus favorable a une dispersion optimale des métaux précieux.

Tableau 2 - Dispersions des phases métalliques $\mathrm{Rh}, \mathrm{Pt}$ et Pd

\begin{tabular}{cccccccc}
\hline \multirow{2}{*}{ Catalyseur } & \multicolumn{2}{c}{$\mathrm{Rh}$} & \multicolumn{2}{c}{$\mathrm{Pt}$} & \multicolumn{2}{c}{$\mathrm{Pd}$} \\
\cline { 2 - 7 } & $\mathrm{CeO}_{2}$ & $\mathrm{CeZr}$ & $\mathrm{CeO}_{2}$ & $\mathrm{CeZr}$ & $\mathrm{CeO}_{2}$ & $\mathrm{CeZr}$ \\
\hline Dispersion (\%) & 88 & 62 & 79 & 78 & 78 & 61 \\
\hline
\end{tabular}

\subsection{Capacitk de stockage en oxygène}

Les mesures de capacite de stockage de l'oxygène ont été effectuées a $400^{\circ} \mathrm{C}$ pour l'ensemble des catalyseurs. Les résultats sont présentés sur la Figure 3.

L'OSC sur I'oxyde mixte cerine-zircone est 4 fois plus importante que celle mesuree dans le cas de la cérine (165 pmol $\mathrm{CO}_{2} \cdot \mathrm{g}^{-1}$ pour $\mathrm{Ce}_{0,63} \mathrm{Zr}_{0,37} \mathrm{O}_{2}$ contre $41 \mathrm{pmol} \mathrm{CO}_{2} \cdot \mathrm{g}^{-1}$ seulement pour $\mathrm{CeO}_{2}$ ). Cette information confirme la nette augmentation de la capacité de stockage en oxygène par substitution des atomes de $\mathrm{Ce}$ par des atomes de $\mathrm{Zr}$. Ce résultat a été attribué a une plus gande mobilité des atomes d'oxygene dans les oxydes mixtes cerine-zircone $[8,9,11]$.

De même, une exaltation de I'OSC est observée lorsque des particules métalliques sont présentes a la surface de I'oxyde. Ainsi I'OSC mesurée a $400^{\circ} \mathrm{C}$ est multipliée par un facteur de 6 en présence de particules de Rh. Suivant ces résultats, les métaux précieux peuvent être classés, selon leur apritude a promouvoir I'OSC, de la façon suivante: $\mathrm{Rh}>\mathrm{Pd}>\mathrm{Pt}$. Cet effet promoteur dépend à la fois de la nature du metal et de la moıphologie des particules métalliques. L'influence relative de ces paramètres est discutée ci-après. 


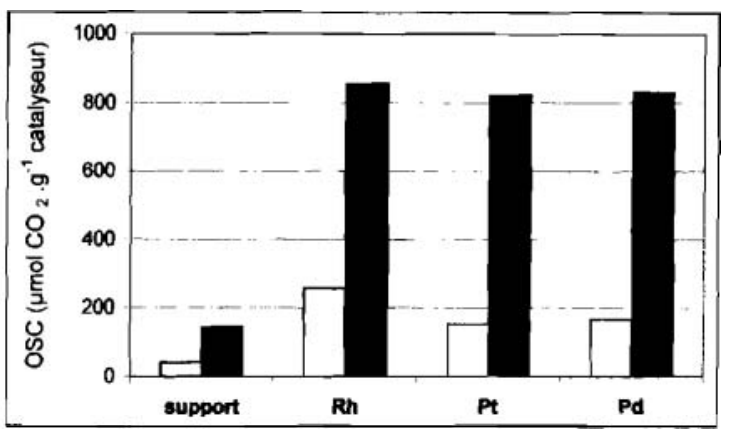

Figure 3 - Evolution de la capacité de stockage en oxygène (OSC)à $400^{\circ} \mathrm{C}$ en fonction de la nature du support ( $\square: \mathrm{CeO}_{2}$, 口: $\mathrm{Ce}_{0,63} \mathrm{Zr}_{0,37} \mathrm{O}_{2}$ ) et du métal (Rh, Pt, Pd)

Afin de déterminer I'influence de la taille des particules métalliques sur le processus de stockage de l'oxygène, les catalyseurs "neufs" ont été traites pendant 4 heures a haute température (entre 800 et $1000^{\circ} \mathrm{C}$ ). Ces catalyseurs sont dits "vieillis". Sous I'effet de la chaleur, les particules de métal - de plus en plus mobiles a la surface de l'oxyde au fur et a mesure que la temperature augmente - migrent et foment des agglomérats de tailles plus importantes. Ce phénomène est connu en catalyse sous le nom de "frittage". Un tel traitement thermique haute température, dit "vieillissement", entraîne donc une modification de la taille des particules metalliques dont l'effet sur I'OSC peut Ctre evalue.

L'OSC des catalyseurs "vieillis" a été mesurée dans les mêmes conditions que les catalyseurs "neufs".Les resultats sont présentés dans le Tableau 3.

Tableau 3 - Influence du vieillissement sur la capacité de stockage de l'oxygène à $400^{\circ} \mathrm{C}$

\begin{tabular}{cccccc}
\hline & \multicolumn{2}{c}{ catalyseurs "neufs" } & \multicolumn{3}{c}{ catalyseurs "vieillis" } \\
\cline { 2 - 6 } & $\begin{array}{c}\text { OSC } \\
\left(\boldsymbol{\mu m o l ~} \mathbf{C O}_{\left.\mathbf{2} \cdot \mathbf{g}^{-1}\right)}\right)\end{array}$ & $\begin{array}{c}\text { Taille de } \\
\text { particules }(\mathrm{A})\end{array}$ & $\begin{array}{c}\text { OSC } \\
\left(\boldsymbol{\mu m o l ~ C O} \cdot \mathbf{g}^{-1}\right)\end{array}$ & $\begin{array}{c}\text { Taille de } \\
\text { particules }(\mathrm{A})\end{array}$ & Vieillissement \\
\hline $\mathrm{Rh} / \mathrm{CeO}_{2}$ & $\mathbf{2 5 7}$ & 10 & $\mathbf{1 4 2}$ & $\mathbf{1 8 1}$ & $\mathrm{H}_{2} / \mathbf{8 0 0}{ }^{\circ} \mathrm{C}$ \\
$\mathrm{Rh} / \mathrm{Ce}_{0,63} \mathrm{Zr}_{0,37} \mathrm{O}_{2}$ & $\mathbf{8 5 8}$ & 15 & $\mathbf{8 3 5}$ & 65 & air $/ 900^{\circ} \mathrm{C}$ \\
$\mathrm{Pt} / \mathrm{CeO}_{2}$ & $\mathbf{1 5 3}$ & 12 & $\mathbf{1 1 6}$ & 25 & air $/ \mathbf{8 0 0} 0^{\circ} \mathrm{C}$ \\
$\mathrm{Pt} / \mathrm{Ce}_{0,63} \mathrm{Zr}_{0,37} \mathrm{O}_{2}$ & $\mathbf{8 2 3}$ & 12 & $\mathbf{5 3 1}$ & 156 & air $/ 1000^{\circ} \mathrm{C}$ \\
$\mathrm{Pd} / \mathrm{CeO}_{2}$ & $\mathbf{1 6 7}$ & 12 & $\mathbf{8 0}$ & 93 & $\mathrm{H}_{2} / 900^{\circ} \mathrm{C}$ \\
$\mathrm{Pd} / \mathrm{Ce}_{0,63} \mathrm{Zr}_{0,37} \mathrm{O}_{2}$ & $\mathbf{8 3 2}$ & 15 & $\mathbf{7 4 9}$ & 17 & air $/ 800^{\circ} \mathrm{C}$ \\
\hline
\end{tabular}

Les resultats, rassemblés dans le Tableau 3, montrent une diminution de la capacité de stockage en oxygene lorsque la taille des particules de métal augmente. Pour le catalyseur $\mathrm{Rh} / \mathrm{CeO}_{2}$ par exemple, I'OSC est pratiquement divisée par deux quand la taille des particules de rhodium varie de 10 a $181 \AA$. Cependant, la capacité de stockage en oxygène ne varie pas linéairement avec la taille des particules metalliques.

Ces résultats confirment le rôle clé joué par la particule métallique au cours du processus de stockage. La particule de metal agit comme une porte d'entrée pour l'oxygène, favorisant ainsi la migration de I'oxygene vers le support. La diminution de I'OSC sous I'effet du frittage de la phase métallique s'expliquerait donc en partie par la diminution du périmètre interfacial metallsupport dans un échantillon constitué de grosses particules par rapport a un échantillon fait de petites particules métalliques bien dispersées.

Enfin nous constatons que le rhodium est le métal le plus efficace quant a la promotion de I'OSC. Comparativement, les catalyseurs $\mathrm{Rh} / \mathrm{CeO}_{2}$ et $\mathrm{Rh} / \mathrm{Ce}_{0,63} \mathrm{Zr}_{0,37} \mathrm{O}_{2}$ présentent les plus grandes capacites de stockage de l'oxygène tant avant qu'après vieillissement. 
Par ailleurs, nous avons étudié I'influence de la température sur la capacité de stockage de l'oxygkne des catalyseurs $\mathrm{Rh} / \mathrm{CeO}_{2}$ et $\mathrm{Rh} / \mathrm{Ce}_{0,63} \mathrm{Zr}_{0,37} \mathrm{O}_{2}$ (Figure 4). Ces résultats montrent clairement l'influence de la nature du support sur I'evolution de I'OSC en fonction de la température. Dans le cas de l'échantillon $\mathrm{Rh} / \mathrm{CeO}_{2}$, I'OSC ne varie que très légèrement avec la tempkrature et atteint un maximum de 296 pmoles $\mathrm{CO}_{2} \cdot \mathrm{g}^{-1}$ catalyseur à $500^{\circ} \mathrm{C}$. A l'inverse, la capacité de stockage du catalyseur $\mathrm{Rh} / \mathrm{Ce}_{0,63} \mathrm{Zr}_{0,37} \mathrm{O}_{2}$ augmente nettement avec la température. Ainsi, I'OSC est multipliée par un facteur 4 entre 200 et $500^{\circ} \mathrm{C}(980$ contre 221 pmoles $\mathrm{CO}_{2} \cdot \mathrm{g}^{-1}$ catalyseur).

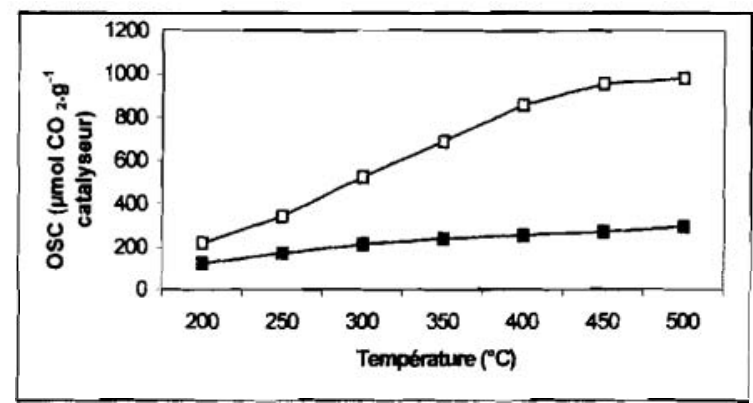

Figure 4 - Evolution de la capacitd de stockage de I'oxygkne $(\mathrm{OSC})$ en fonction de la temperature $\left(\square: \mathrm{Rh} / \mathrm{CeO}_{2}\right.$, $\left.\square: \mathbf{R h} / \mathrm{Ce}_{0,63} \mathrm{Zr}_{0,37} \mathrm{O}_{2}\right)$ - influence du support

$\mathrm{Au}$ vue de ces résultats, la question de l'origine d'une telle différence de comportement en température se pose. Sachant par ailleurs que les atomes d'oxygene sont beaucoup plus mobiles sur les oxydes de type cérine-zircone que sur la cérine, nous proposons que le processus de stockage de I'oxygene serait limite a la surface de l'oxyde dans le cas de la cérine et qu'il impliquerait la participation d'atomes d'oxygene du cceur de I'oxyde dans le cas du catalyseur $\mathrm{Rh} / \mathrm{Ce}_{0,63} \mathrm{Zr}_{0,37} \mathrm{O}_{2}$. Afin de vérifier cette hypothese, le nombre total d'atomes d'oxygene a la surface de chacun de ces oxydes a été calculé comme précédemment decrit [11]. Les deux oxydes $\mathrm{CeO}_{2}$ et $\mathrm{Ce}_{0,63} \mathrm{Zr}_{0,37} \mathrm{O}_{2}$ sont de structure cubique. Les paramètres de maille $\mathrm{a}=\mathrm{b}=\mathrm{c}$ sont respectivement de 5.4113 et $5.3044 \AA$. En fonction de la face cristalline considérée, la densité surfacique en atomes d'oxygene est donnée par les relations suivantes

- face (100) : 4 atomes situés à une distance $\frac{a}{4}$ du plan (100) dans une surface de $a^{2}$

- face (110) : 4 atomes situés dans le plan (110) dans une surface de $a^{2} \sqrt{2}$

- face (111): 2 atomes situés a une distance $\frac{a}{3 \sqrt{3}}$ du plan (111) dans une surface de $\frac{a^{2} \sqrt{3}}{4}$

Nous supposons par ailleurs que (i) seuls les atomes d'oxygene liés a des atomes de cerium participent au processus de stockage de I'oxygkne, (ii) que la composition de surface de l'échantillon est identique a la composition globale de l'échantillon, c'est-a-dire qu'il n'y a pas de ségrégation superficielle du zirconium et (iii) que seul un atome d'oxygene sur quatre est impliqué dans le processus de stockage. Le nombre maximum d'atome d'oxygkne potentiellement "mobilisables"pour I'OSC est alors donné par :

$\mathrm{O}_{\text {réductibles }}=\mathrm{O}_{\text {surface }} \times \frac{\mathrm{Ce}}{4}$

En considerant une équi-répartition des plans cristallographiques (100), (110) et (111), le nornbre d'atomes d'oxygkne "réductibles" a été calculé (Tableau 4). 


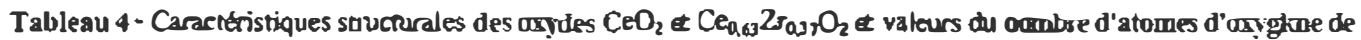
surnine et du nombre d'atomes d'oxygene "neductibles"

\begin{tabular}{|c|c|c|c|c|}
\hline Oxyde & $\begin{array}{l}\text { Parametre de maille } \\
(\AA)\end{array}$ & $\begin{array}{l}\text { Nombre d'atomes } 0 \\
\text { parnm de suŕace }\end{array}$ & $S_{\text {HET }}\left(m^{2} \cdot 8^{-1}\right)$ & $\begin{array}{c}\mathrm{O}_{\text {rtductibles }} \\
\left(\mathrm{pmol} \text { atomes } \mathrm{O} \cdot \mathrm{g}^{-1}\right)\end{array}$ \\
\hline $\mathrm{C}_{\mathrm{cO}}$ & 5.4113 & 13,1 & 25 & 136 \\
\hline $\mathrm{Ce}_{0,63} \mathrm{Zr}_{0,37} \mathrm{O}_{2}$ & 5,3044 & 13.5 & 43 & 152 \\
\hline
\end{tabular}

Dans le cas de l'oxyde cérine seul (en l'absence de metal). rOSC à $500^{\circ} \mathrm{C}$ est de 64 umol. $\mathrm{g}^{-1}$. Seule la moitié des atomes d'oxygene de surface (136 بmol. $\left.\mathrm{g}^{-1}\right)$ potentiellernent "disponibles" sont impliques. En revanche. I'OSC a $500^{\circ} \mathrm{C}$ de l'oxyde mixte $\mathrm{Ce}_{0,63} \mathrm{Z}_{\mathrm{r} 0}, \mathrm{O}_{2}$ est de $480 \mu \mathrm{mol} .8^{-1}$. Dans ce cas, le processus de stockage a lieu bien au-delà de la premil-re couche d'atomes d'oxygkne. Nous pouvons d'ailleurs calculer le nombre de conches d'atomes d'oxygene impliquées dans le processus de stockage de l'oxygène. Ce nombre est dome par le rapport entre IOSC de l'oxyde et le nombre d'atomes d'oxygene "réductibles" (14):

$\mathrm{NC}=\frac{\text { USC }_{\text {oxyde }}}{\text { Oréductioles }_{\text {res }}}$

Sachant que : $\mathrm{OSC}_{\text {oxyde }}=\mathrm{OSC}_{\text {mesurte }}-\mathrm{OSC}_{\text {metel }}$

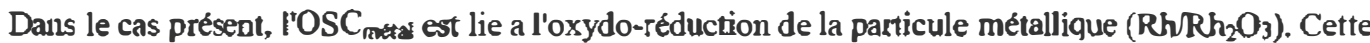
grandeur est fonction de la nature du métal et de la teneur en métal. Les resultats des calculs sont présentés dans le Tableau 5 .

Tableau 5 - Nombre de couches d'stomes $O$ inpliquées dans le stockage de Foṣgghe

\begin{tabular}{|c|c|c|c|c|}
\hline & OSC $C_{\text {mant }}$ & USC & $U_{\text {ntmang }}$ & NC: \\
\hline $\mathrm{CeO}_{2}$ & 64 & 0 & 136 & 0,5 \\
\hline $\mathrm{Rs} / \mathrm{CeO}_{2}$ & 296 & 115 & 136 & 1,3 \\
\hline $\mathrm{Ce}_{0,63} \mathrm{Zr}_{0,37} \mathrm{O}_{2}$ & 480 & $\mathbf{0}$ & 152 & 3,1 \\
\hline $\mathrm{Rh} / \mathrm{Ce}_{0,63} \mathrm{Zr}_{0,37} \mathrm{O}_{2}$ & 980 & 128 & 152 & 5,6 \\
\hline
\end{tabular}

Ces résultats confirment que le stockage de l'oxygkne est limite a la surface de la cérine seule alors qu'il implique la participation de plusieuss couches d'atomes d'oxygene du ccetr de l'oxyde dans le cas de l'oxyde mixte cerine-zircone. La reduction a couur de l'oxyde $\mathrm{C}_{0.63} \mathrm{Zr}_{0.37} \mathrm{U}_{2}$ est donc trés clairement a l'origine de l'impononte augmentation, en fonction de la temperature, de la capacité de stockage de

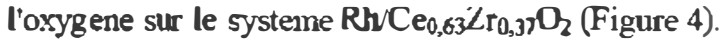

Schématiquement, le processus de stockage de I'oxygkne pourrait ètre décrit de la façon suivante (Figure5) :

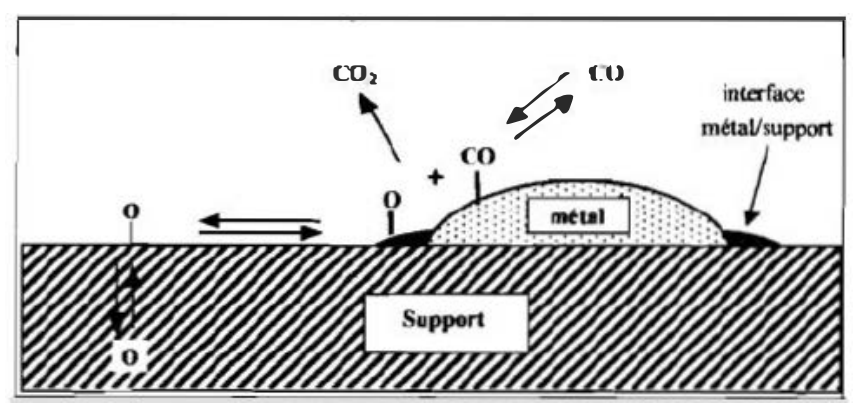

Figure 5 - Schkma du mbanisme de stockage de roxnghoe 
La molecule de CO s'adsorbe sur le métal et réagit avec un oxygbne de la surface de I'oxyde. La formation de $\mathrm{CO}_{2}$ résultant de cette réaction implique la formation d'une lacune d'oxygbne sur le support. Sur I'oxyde de cerium seul, ce processus se poursuit jusqu'a reduction complete de la surface. Dans le cas de I'oxyde $\mathrm{Ce}_{0,63} \mathrm{Zr}_{0,37} \mathrm{O}_{2}$ notamment, du fait de la grande mobilité de I'oxygene au sein des oxydes mixtes, un oxygbne d'une couche inteme peut diffiser a la surface de I'oxyde et combler la lacune formée au cours de la première étape. Cet oxygène peut alors réagir à son tour avec une nouvelle molécule de $\mathrm{CO}$ pour former une nouvelle molécule de $\mathrm{CO}_{2}$ et créer une nouvelle lacune d'oxygene a la surface...

\section{CONCLUSIONS}

L'étude de catalyseurs du type métaux précieux ( $\mathrm{Rh}, \mathrm{Pt}$, Pd) supportés sur $\mathrm{CeO}_{2}$ et $\mathrm{Ce}_{0,63} \mathrm{Zr}_{0,37} \mathrm{O}_{2}$ a démontré tout l'intérêt de telles formulations en catalyse trois-voies. Ainsi, la substitution du zirconium dans le réseau de la cerine permet d'améliorer simultanément (i) la stabilité thermique de I'oxyde et (ii) la capacité de stockage de l'oxygene $\left(165 \mu \mathrm{mol} \mathrm{CO}_{2} \cdot \mathrm{g}^{-1}\right.$ pour $\mathrm{Ce}_{0,63} \mathrm{Zr}_{0,37} \mathrm{O}_{2}$ contre $41 \mu \mathrm{mol} \mathrm{CO}_{2} \cdot \mathrm{g}^{-1}$ pour $\mathrm{CeO}_{2}$ ). Par ailleurs, une exaltation de I'OSC est observée en presence de particules metalliques à la surface de l'oxyde. De tels ilôts métalliques agiraient comme des portes d'entrée pour la migration ultérieure de I'oxygene vers le support. Aussi, toutes modifications de la morphologie de ces particulesde metal provoquent une variation de la capacite de stockage. En effet, pour I'ensemble des catalyseurs étudiés, I'OSC diminue après vieillissement. Cependant aucune relation directe n'a pu être etablie entre la diminution de I'OSC et I'augmentation de la taille des particules metalliques. Enfin, l'évolution de la capacite de stockage de I'oxygene en fonction de la température révèle que (i) dans le cas des systemes supportés sur cérine I'OSC se limite a une réduction complbte de la proche surface du support et que (ii) le stockage de l'oxygene au sein des echantillons cérine-zircone implique la réduction du cœur de I'oxyde. Dans les deux cas la cinetique du processus de stockage est contrôlée par la diffusion de l'oxygene a la surface et au cœur du matériau.

\section{REFERENCES}

1. A. Trovarelli, Cat. Rev. Sci. Eng., 38 (1996) 439-520

2. H.C. Yao, Y.F. Yu Yao, J. Catal., 86 (1984) 254-265

3. T. Miki, T. Ogawa, M. Haneda, N. Nakuta, A. Ueno, S. Tateishi, S. Matsuura, M. Sato, J. Phys. Chem., 94 (1990) 6464-6467

4. A. Trovarelli, F. Zamar, J. Llorca, C. De Leitenburg, G. Dolcetti, J.T. Kiss, J. Catal., 167 (1997) 490 520

5. C.E. Hori, H. Permana, K.Y. Simon Ng, A. Brenner, K. More, K.M. Rohmoeller, D. Belton, Appl. Catal. B, 16 (1998) 107-117

6. S. Rossignol, Y. Madier, D. Duprez, Catal. Today, 50 (1999) 261-270

7. E. Rocchini, A. Trovarelli, J. Llorca, G.W. Graham, W.H. Weber, M. Maceijewski, A. Baiker, J. Catal., 194 (2000) 461-478

8. J. Kaspar, P. Fornasiero, M. Graziani, Catal. Today, 50 (1999) 285-298

9. J-P. Cuif, G. Blanchard, O. Touret, A. Seigneurin, M. Marczi, E. Quéméré, SAE Technical Papers Series, 970463 (1997)

10. S. Kacimi, J. Barbier Jr, R. Taha, D. Duprez, Catal. Lett., 22 (1993) 343-350

11. Y. Madier, C. Descorme, A.M. Le Govic, D. Duprez, J. Phys. Chem. B, 103 (1999) 10999-11006 